\title{
EDITORIAL
}

\section{RELEVANCE OF CONTROLING FOR CONFOUNDING IN OBSERVATIONAL STUDIES}

Authors from Editors of Archivos de Bronconeumología

${ }^{1,2}$ Esther Barreiro, ${ }^{2,3}$ Xavier Muñoz, ${ }^{2,4}$ Francisco Javier González-Barcala, ${ }^{5}$ Víctor Bustamante, ${ }^{6}$ Ignacio de Granda

${ }^{1}$ Pulmonology Department-Muscle Wasting and Cachexia in Chronic Respiratory Diseases and Lung Cancer, IMIM-Hospital del Mar, Barcelona, Spain

${ }^{2}$ Centro de Investigación en Red de Enfermedades Respiratorias (CIBERES), Instituto de Salud Carlos III (ISCIII), Barcelona, Spain.

${ }^{3}$ Servicio de Neumología Hospital Vall d'Hebron Barcelona

${ }^{4}$ Pulmonology Department-Hospital Clinico Universitario de Santiago de Compostela

${ }^{5}$ Servicio de Neumología, Hospital Universitario Basurto. Bilbao. Osakidetza. Departamento de Medicina. Universidad del País Vasco.

${ }^{6}$ Servicio de Neumología, Hospital Universitario 12 de Octubre, Universidad Complutense. Madrid

Corresponding author: Esther Barreiro, e-mail: ebarreiro@imim.es

CONFLICT OF INTEREST: None

Word count: 489 


\section{EDITORIAL}

In the Annals of the American Thoracic Society (Ann Am Thorac Soc) Journal an article on the control of confounding and reporting of results in causal inference studies has been recently published (1). This publication was the result of the need of establishing a guidance document as viewed by the 47 editors of 35 respiratory, sleep, and critical care journals who participated in the publication of the article (1). The objective of the current editorial is to provide a brief outline on the most relevant issues analyzed in that article (1).We expect that this editorial will be informative for researchers, authors, and peerreviewers on the most relevant features to be taken into consideration at the time of designing and reporting results in observational studies.

The relevance of causal inference relies on the fact that associations can be made to establish the causal effect of a given exposure on an outcome (1). Causal inference is commonly used to answer etiologic questions in a study. These are relevant investigations as they help elucidate clinical problems. However, caution must be taken when designing these studies as confounding, an unclear hypothesis, and selection biases may interfere with the study outcomes, which may jeopardize interpretation of the results.

A confounder has been classically defined as a third variable that is associated with the target exposure, is a cause of the outcome of interest, and is not located in the causal pathway between the exposure and the outcome (2). Despite that this classical definition of a confounder has been accepted in the guidance document (1), causal models are recommended with the aim to identify confounders at the time of designing observational studies (2-4). Examples of graphs representing causal models are provided in the guidance document for those interested in learning more about them (1).

In the article (1), reference to several approaches that authors tend to do in order to control for confounding has been made. As such it has been sufficiently argued that 
variable selection methods do not help control for confounding as they may ignore the underlying cause inherent to the study hypothesis (1). Furthermore, emphasis has also been made on avoiding interpretation of causal inference on the basis of $P$ value, since it does not provide any information on the magnitude, direction or clinical relevance of the associations between variables (1).

In summary, in the guidance document recently published (1) it is recommended that observational (cohort, cross-sectional, and case-control) studies adhere to the STROBE (Strengthening the Reporting of Observational Studies in Epidemiology) statement, especially at the time of presenting the results in tabular form (5). We, Editors of Archivos de Bronconeumología, agree with these recommendations and for the sake of clinical research will ensure that the results of the observational studies published in our journal adhere to them. We would also like to invite our authors to read the guidance document published in the January issue of the Ann Am Thorac Soc.

\section{Reference List}

1. Lederer DJ, Bell SC, Branson RD, Chalmers JD, Marshall R, Maslove DM, et al. Control of Confounding and Reporting of Results in Causal Inference Studies. Guidance for Authors from Editors of Respiratory, Sleep, and Critical Care Journals. Ann Am Thorac Soc. 2019;16(1):22-8.

2. Greenland S. Quantifying biases in causal models: classical confounding vs colliderstratification bias. Epidemiology. 2003;14(3):300-6.

3. Hernan MA, Hernandez-Diaz S, Robins JM. A structural approach to selection bias. Epidemiology. 2004;15(5):615-25.

4. Williamson EJ, Aitken Z, Lawrie J, Dharmage SC, Burgess JA, Forbes AB. Introduction to causal diagrams for confounder selection. Respirology. 2014;19(3):303-11.

5. von EE, Altman DG, Egger M, Pocock SJ, Gotzsche PC, Vandenbroucke JP. The Strengthening the Reporting of Observational Studies in Epidemiology (STROBE) statement: guidelines for reporting observational studies. Ann Intern Med. 2007;147(8):573-7. 